\title{
Zinc Deficiency and Association with Cognitive Performance in School-aged Children: Peer Review
}

\author{
Gomaa F Salma* \\ Institute of Public Health and Human Ecology, American University in Cairo, Egypt \\ *Corresponding Author: Gomaa F Salma, Institute of Public Health and Human \\ Ecology, American University in Cairo, Egypt.
}

Received: August 20, 2021

Published: September 30, 2021

(C) All rights are reserved by Gomaa F Salma.

\begin{abstract}
In developing countries, micronutrient deficiencies are of major impact on children development. Zinc is among the micronutrient which is supposed to play an important role in children development, including both growth and cognitive performance. Although the evidencefortheroleofzincin cognitivedevelopmentthoughanimals'studiesis proved, evidence from human studies is still not conclusive. The association between dietary zinc status and cognitive functions through epidemiological studies, remains elusive. This review is summarizing current literature and epidemiological studies that investigated the association between zinc intake, through diet or supplement, zinc status (plasma/serum zinc concentration), and the different domains of cognitive functions in school-aged children.
\end{abstract}

Keywords: Zinc; DNA Synthesis; Plasma

\section{Introduction}

The scientific understanding for the exact role of zinc as an important micronutrient in many biological processes as well as the significance of zinc deficiency in public health has been intensively well-established through the past few decades. It was not until 1960 since zinc was recognized as an essential micronutrient of unique biological functions in human's physiology. Nowadays, it is confirmed that zinc plays an important role in number of physiological activities in the body, including gene expression, enzymatic reaction, protein and DNA synthesis, cell development, immune function, as well as cellular metabolism. Zinc is important for the activity of over 300 enzymes that aid in metabolism, digestion, nerve function and many other processes. Owing to its unique role, zinc is thus essential for optimal growth, immunocompetence, as well as visual acuity $[1,2]$.
It has been estimated that $30 \%$ of the world population are zinc deficient. Zinc deficiency occurs in individuals and populations whose diets are low in sources of bioavailable zinc (such as red meat products and seafood) and high in unrefined cereals (rich in phytate and dietary fiber). These dietary patterns are widely common in developing countries. That is why zinc deficient, as a public health issue, is well demonstrated in developing countries, affecting growth and development of children [3]. Zinc is present in many organs and body fluids, including the brain, where it contributes to its structure and function [1]. Evidence from animal and human studies suggests that its deficiency may lead to impaired cognitive development.

The objective of this narrative review is to (1) summarize current epidemiological studies, both Observational and interventional, that investigated the association between zinc intake, through diet and/or supplementation, zinc status (plasma/serum zinc

Citation: Gomaa F Salma. "Zinc Deficiency and Association with Cognitive Performance in School-aged Children: Peer Review". Acta Scientific Medical Sciences 5.11 (2021): 182-188. 
concentration), and the different domains of cognitive functions in school-aged children. (2) The review also aims to highlight the gaps in current literature and suggest avenues for future research to be fulfilled. This article is organized as follows: The next section presents the methodology adopted to conduct this narrative literature review, followed by the sections summarizing the outcome of literature review; and the last section concludes the paper with suggestions on potential avenues for future research.

\section{Research Methodology}

Inclusion criteria

Author included manuscripts for observational studies (cross sectional studies) that tackled the association between zinc status (in the form of plasma/serum zinc concentration) and any of the three domains for cognitive performance (motor, intelligence, motor skills and executive functions) Also, the author included randomized clinical trials that studied the effect of zinc intervention (through diet or supplementation) on the domains of cognitive functions. The inclusion criteria were limited to cross sectional and randomized clinical trial done cross the world. Also, the population of interest was limited to school-age children, were studies related to infants were excluded. The author also included narrative review articles.

\section{Literature identification}

Author started the literature search by searching for the key words "zinc status in children", "Zinc status and cognitive performance", "zinc deficiency and cognitive performance", "zinc deficiency in children", "zinc supplementation in children" "zinc observational studies in children". For each manuscript, preliminary relevance was determined by title. From the title if the content seemed to discuss the association between zinc status and cognitive performance in children, then the author obtained its full reference (author, year, title, and abstract, for further evaluation). The author searched Google scholar, web of science, Science direct and ProQuest. The search was limited for the publication date between 1998 and 2020. (Manuscript published in the past 22 years), to build the review on the recent literature. After initial screening of the titles, a total of 35 studies were identified as potential studies relevant to the research topic.

\section{Screening for inclusion}

The author read the abstracts of the studies to further identify their relevance to the research Topic. A total of 25 studies were considered relevant and author obtained the full-text article for quality assessment.

\section{Quality and eligibility assessment}

The author went through the full-text articles to further evaluate the quality and eligibility of the studies. The author considered journal articles published by reputable publishers as high-quality research, and therefore, included them in the review. On-line presentations and reports were excluded from the review due to lack of peer-review process. After careful review, a total of 8 studies were excluded because they tackled children with special needs, where 7 studies were tackling zinc deficiency in school aged children with attention deficit hyperactivity disorder (ADHD), and One study explored zinc deficiency in lead-exposed schoolchildren.

\section{Definition of cognitive development}

Cognition is a complex model that consists of a sequence of processes through which person can registers, encodes, selects, maintains, transforms, stores, and retrieves information. Such processes include visual and sensorial aspects as well as thinking, memory, and learning. The cognitive performance can be sub divided into domains, according to the type of processes it includes. Such domains are intelligence, motor skills and executive functions. The later domain is further subdivided into memory, attention, language, and global cognitive function [2-4]. In the first year of human life, child learns to construct mental structures. Such structures greatly depend on child's body movements. As the infant grow with time, he continuously develops his attention skills by exploring and focusing on the new aspects of his environment. Later, in his pre-school years, he extends his skills to further concentrate on testing and organizing the information $[2,4,5]$.

\section{Cognitive function in children and adolescence}

Throughout the school years, cognition continues to develop and mature as a set of solid operations, including skills like thought, memory, and language development. During this time of growth, multiple stimuli can be understood simultaneously, and attention abilities increase, reflected in greater learning and memory. By the time of adolescence, adult intelligence and hypothetical thinking are fully developed. Assessment of cognitive development would include evaluation of perception, thought, attention, memory, language, and activity, such concepts which reflect the three main domains of cognition [2-4]. 
Biological mechanisms of zinc deficiency on cognitive development

Zinc is abundant in brain tissues, particularly the hippocampus, the area participating in learning and memory, in addition to the amygdala, striatum and neocortex. Zinc is not only important for the function of the brain, but being pounded to brain's protein, reflect that it is also important for the brain structure as well $[1,2]$. Evidence indicates that there are number of mechanisms by which zinc deficiency can affect with development including, (1) zinc is a key modulator of intracellular and intercellular neuronal signaling. This function is supported by that fact that zinc is presence in high concentrations in the synaptic vesicles of the special neurons, called the 'zinc containing' neurons, in the forebrain. During synaptic events, zinc actas a neurotransmitter, released and diffuses into postsynaptic neurons. Zinc as well function in biochemical processes like myelination and release of other neurotransmitters like g-amino butyric acid [GABA] and glutamate [2,3,5]. (2) Since Zinc is essential for the activity of many metalloenzymes, cellular functions including protein, and DNA synthesis, cellular growth, differentiation, and metabolism, thus zinc deficiency may hinder these processes and thus alter subsequent development [3,5], (3) There is also some evidence to suggest that zincdeficiency results in lowered levels of long omega- 3 and omega- 6 fatty acid chains, which may cause impaired fatty acid metabolism in the neurons [2], (4) In addition, zinc is involved in the metabolism of thyroid hormones, receptor function and transport of other hormones that could affect the central nervous system [2]. All these evidences support that zinc is important for neurogenesis, neuronal migration, and synaptogenesis. Consequently, zinc deficiency could alter the process of neurotransmission and all the subsequent neurophysiological development depending on it [2]. Most of the evidence for the effect of zinc deficiency on the central nervous system are acquired mainly from animal studies. Experimental Studies in rats have showed that zinc deficiency is associated with structural malformations of the brain, such as reduced cerebellum size, anencephaly, microcephaly, and hydrocephaly, during the early stages of brain development. In the later stages of brain development, results have showed that zinc deficiency causes behavioral disorders and reduced brain functions, such as reduced activity, deficits in short term memory, poor attention, and spatial learning. Also, studies showed that zinc deficiency had a negative impact on motor activities. When it comes to human, Evidence is less clear [1,3].
Evidence linking zinc status and cognitive performance in school aged children

\section{Observational studies}

The current scientific research revealed the positive impact of zinc through the preschoolyears of children, however, the evidence from studies in school-age children is controversial [6,7]. some observational studies among elementary school-age children showed that there is a positive correlation between hair zinc and reading ability, suggesting that zinc deficiency has a role in academic performance [6]. In India, a cross-sectional observational study was done to evaluate the effect of zinc and Iron deficiency on short term memory of children in the age (6-11 years), from an urban corporation school. All children with iron and zinc deficiency showed memory deficits [7]. On the other hand, in a cross-sectional study conducted in school-age, stunted children in Guatemala, it was reported that there were no differences in attention span based on zinc status of children, as measured by subtests of the Detroit Tests of Learning Aptitudes [6]. When it comes to adolescence, A crosssectional study was conducted to examine the association between plasma zinc (PZ) and erythrocyte zinc (EZ) levels with cognitive performance in Indian adolescent girls. Cognitive performance was assessed by simple-reaction-time (SRT), recognition-reaction-time (RRT), visual-memory, Raven's Progressive Matrices (RPM) test. Results showed that Zinc deficiency in adolescent girls was associated with poor cognition performance, demonstrated by the negatively associated between PZ and EZ with SRT and RRT, and the positively association with Memory and RPM [8]. The same conclusion has been obtained in a similar study done on high school female students in Iran.In this cross-sectional study, each participant completed a 3 day 24-h food recall questionnaire to assess the daily zinc intake. Also, zinc status was assessed by measuring serum zinc level. Cognitive function was obtained by summing the scores of five tests including Raven's Standard Progressive Matrices test (RPM) on non-verbal intelligence, Benton visual retention test, Wechsler memory scale, Bonnardel accuracy test and letter eliminating test. Results showed that there is a strong positive association between serum zinc level and Raven's, memory, Bonnardel's and letter eliminating scores, however, there was no association with dietary zinc intake [9].

\section{Experimental studies}

Although observational studies can provide useful information about zinc deficiency, results are more susceptible to confounding 
by other factors that can occur along with zinc deficiency and are also associated with impaired cognitive functions in children, such as poverty and other nutritional deficiencies. Thus, RCTs are important to identify the exact effect of zinc deficiency on children's cognitive performance [5]. The RCTs related to the effect of zinc supplementation, on cognitive development among school-age children, can be divided according to the cognitive domain (executive functions, motor skills, and intelligence) under evaluation.

\section{Zinc intervention and executive function in children}

The executive domain of cognitive function mainly refers to the attention, inhibitory control, memory, and language. A randomized clinical study was done on adolescence girls in India to test the efficacy of zinc supplementation on cognitive function. Zinc was intervened through diet (zinc-rich food), or ayurvedic zinctablet. Cognitive assessment used in evaluation was the simple reaction time (SRT), recognition reaction time (RRT), visual memory, and Raven's Progressive Matrices (RPM). It was reported in this study that Supplementation of ayurvedic zinc and zinc-rich foods was effective in improving cognitive performance in adolescence girls. This was reflected in the significant increase in the scores for memory and RPM for the intervened groups vs control as well as a significant decrease in the Mean SRT and RRT [10]. In contrast, Gibson., et al. conducted a zinc supplementation study in stunted school-age boys, within 5-7 years of age, In Canada. Cognitive assessment used in evaluation was the 4 subtests from the Detroit Tests of Learning Abilities The study extended for 12 months, at the end of the which it was reported that zinc supplementation had no significant impact on attentionspan [11].

\section{Zinc intervention and intelligence in children}

A randomized, double-blind, placebo-controlled field trial was carried out in public elementary school in Mashhad- Iran to investigate the effect of Zinc intervention on cognition performance of 45 healthy children, aged 6-8 years. The cognitive test used in the trial was Raven IQ (Intelligence Quotient) test. The study extended for 6 months at the end of which it was reported that the intellectual development in the intervened groups was significantly higher as compared to the control [12]. On the other hand, Cavan., et al. reported that zinc supplementation in children had no significant effect on the general mental abilities, confirmed by the cognitive score of the Detroit tests of learning aptitude, although children did respond to zinc supplementation with significant changes such cognitive scores. This result agrees with the results of the study by Gewa., et al. conducted in children in Kenya in which children's diets were supplemented with meat in order to increase their bioavailable zinc intake. The cognitive assessment used in the trial was Raven IQ test (Intelligence Quotient). The study extended for 2 years, at the end of which, there were no significant differences in Raven test scores between the children consuming intervention diet with meat, compared with control participant (consuming the control diets) [3]. In Indonesia, similar conclusion was obtained by Retno., et al. who reported that Zinc supplementation produced highly positive responses in weight for age and heightfor age increments in children with zinc deficiency compared with placebo. However, there was no significant impact on cognition measured by intelligence quotient (IQ) [13].

A number of follow up studies was investigating the impact of zinc supplementation during infancy on the intelligence indices during childhood. One of these follow-up studies reported that zinc supplementation for infants from 12 to 35 months, resulted in no significant improvements in intellectual function scores in the zinc supplemented group compared with the placebo control group, when children were followed up at 7-9 years of age. A study of similar design was conducted in India, at which zinc supplementation was given to infants from 4-6 month for 6 months. The cognitive assessment test used in the study was the Wechsler IntelligenceScaleforChildren,theRaven'sColoredProgressiveMatrices (CPM), and school performance tests. The study participants were assessed 8 years later, in terms of the intelligence indices, and the results showed that there were no long-term effects of zinc supplementation given for infants, on their intelligence indices during their childhood $[3,14]$.

\section{Zinc intervention and motor skills in children}

In an evaluation of 372 children (ages 6-9) from low-income families in China, Penland., et al. reported that children who received zinc intervention with or without other micronutrients for 10 weeks, functioned better on all subtests of the CPAS-R battery (Cognition Psychomotor Assessment-Revised battery) than did those who were intervened with micronutrients only. These results are agreeing with the results obtained by Sandstead., et al. who showed that zinc intervention with other micronutrients significantly improved neuropsychologic performance including the tasks of tapping, circular tracking (motor) and oddity (concept 
formation) compared with micronutrients alone [3]. Another trail that was done in Chinese children and Mexican American children from Texas have found that children who received zinc intervention proved superior neuropsychological performance, particularly in reasoning, when compared with controls [5]. In contrast, Hubbs-Tait., et al. reported a negative association between plasma zinc concentration and motor scores of the motor subset within the McCarthy scales of children's ability test [3].

Based on current literature, evidence is suggesting that zinc supplementation is having a significant positive impact on the cognitive performance in school-aged children that is mainly dominant on specific neuropsychological processes that include time-dependent challenging tasks, such as attention and reasoning. However, it could be of limited impact on the general performance tasks $[5,6]$. However, more studies are needed to replicate existing data [5].

\section{Prenatal zinc intervention and cognitive performance}

Some studies have investigated the effect of zinc supplementation during pregnancy (prenatal) on the cognitive performance of the off springs during childhood. Most of the reported studies did not show a significant impact for prenatal intervention on the cognitive performance during childhood. Laura., et al. reported that maternal gestational zinc supplementation was having no effect of different aspects of child development at 54 months of age in Peru [15]. The same conclusion has been obtained by parul., et al. in-cohort study that took place in Nepal. In such study, the intellectual and motor functions of 676 children, whose mother participated in an RCT of micronutrient supplementation during pregnancy, including zinc, was assessed. It was reported that for the group intervened with zinc the difference across outcomes was not significant [16]. Furthermore, Tsunenobu., et al. also evaluated the effect of prenatal zinc supplementation on neurological development, mainly mental and psychomotor development, of 355 children whose mothers participated in an RCT of zinc supplementation, received in the latter half of pregnancy period. Results showed that the intervention had no effect on the neurologic development of such children at age 5 years [17].

\section{Meta-analysis and systematic reviews}

In 2015, a systematic review and A meta-analysis study was performed to examine the association between zinc intake and/or zinc status with cognitive function domains in children and adults
[3]. Of all the potentially available studies, a meta-analysis study was conducted for six RCTs (that were eligible for meta-analysis) done in children that examined the effect of zinc supplementation either pre- or post-nataly. The analysis revealed that the overall pooled standard mean difference of the impact of zinc intake on the three domains of cognitive functions (intelligence, executive function, and motor skills) was not significant. The authors have interpretated the lack of significant effect due to: (1) The sample size was considerably small. Only a small number $(n=6)$ of studies was eligible to be included in this meta-analysis. This is because of the lack of data that could be included because of the difference in thestudy design and the type of cognitive test applied per each cognitive domain. In other words, heterogeneity in the study designs was the main constraint for including more studies in the analysis. (2) Another reason is that many of the studies included in this meta-analysis were assessed as having moderate-to-high risk of bias, which may have impacted pooled results outcome. Authors were unable to restrict meta-analyses to studies at low (or lower) risk of bias, or to stratify studies according to risk of bias because of the limited number of studieseligible for meta-analysis [3].

Inspite of the fact that the presented Meta-analysis failed to show a significant effect of zinc supplementation on cognitive domains in children, taken as a whole, there were some small indicators of improvement on some aspects of executive function and motor development associated with zinc supplementations [3].

A very important conclusion obtained for the meta-analysis is that when the data was Stratified by subgroups based on whether the child was given the intervention directly or given prenatally to the mother, results revealed that prenatal supplementation (maternal supplementation during pregnancy), did not have a longterm impact on cognitive performance of the offspring during their childhood. On the other hand, RCTs at which the supplementation was given directly to the child, showed that there is a significant effect of zinc intake on some aspects of executive function and motor development. However, this must be interpreted with caution because of the limited number of studies included in these analyses [3].

\section{Conclusion and Future Perspective}

A considerable number of studies have been done in different regions across the world to assess the effect of zinc status and/or 
zinc supplementation on school-aged children's cognitive performance. However, the outcome of this studies has yielded inconsistent conclusions about the impact of zinc deficiency. Some studies have reported a positive effect of zinc intake/ status on different cognitive domains, other studies have reported inconsistent or mixed results. This can be attributed to number of factors. Some of these factors could be related to theindividual subjects recruited in the studies, such as (1) differences in the pre-existing zinc status of the study participant, (2) the current content and bioavailability of zinc in their local diets, (3) and in the incidence of common infections that can affect children growth independently of the zinc status. Other factors could be related to the methodological aspects and study designs, such as differences in the dose, in the method of administration, or in the duration ofsupplementation (in RCTs). All these factors could have affected their results. Also, in some cases, the sample sizes may have been insufficient to identify significant differences with statistical confidence [1]. Inspite of the facts that the current meta-analysis conducted on some of these studies showed no significant overall effect of zinc supplementation on any of the assigned cognitive aspects, a positive effect of zinc supplementation on cognitive function cannot be ignored [3]. The meta-analysis reported and highlighted the differences between the studies available, to declare that it is not possible to compare what is very different. It also highlighted the fact that there is limited number of standardized studies that eligibility criteria to be included in the meta- analysis [1,3]. Consequently, the insufficient evidence is mainly because of the lack of well-designed and carefully controlled long-term RCTs that can deeply investigate the relationship between zinc intake and/or status, and cognitive functions in school-aged children. Studies should be reported in a consistent and standardized manner as well as in a comparable measuring unit to enable future and accurate comparisons of the outcome data with other studies [3]. Another point that needs to be tackled in future research is the combined role of zinc and other micronutrient in enhancing cognitive performance in school-aged children. Most of the current studies that are linking zinc to child development has not addressed the possibility of interactions with other micronutrient deficiencies. Such hypothesis is supported by the fact that animal products, which are the primary source of zinc, are also important sources of other micronutrients including iron and vitamin B-12, which suggests that children who are zinc deficient are most probability deficient in iron and vitamin B-12. At the same time, the three micronutrients have been associated with deficits in cognitive performance. Thus, additional studies are required to address the effects of zinc status/zinc intake in combination with other micronutrients to identify the possibility of synergetic Interaction between micronutrients [2]. Another point that needs to be tackled also in future research is the sex differentiation with zinc deficiency. Sex differences have been found in several studies, assuming that males are more vulnerable to zinc deficiency than females. However, additional research is needed to confirm and this finding and to examine the hypothetical mechanisms for such sex differences [6]. Last but not least, Additional research is required to determine the exact biological mechanisms, the critical periods in children life at which they could be more vulnerable to the negative effect of zinc deficiency, the threshold of severity as well as the long- term effects of zinc deficiency on cognitive development [3].

\section{Competing Interest Statement}

No potential competing interest was reported by the author.

\section{Bibliography}

1. Nissensohn M., et al. "Effect of Zinc Intake on Mental and Motor Development in Infants: A Meta-Analysis". International Journal for Vitamin and Nutrition Research 83 (2013): 203215.

2. Bhatnagar S and Taneja S. "Zinc and cognitive development". British Journal of Nutrition 85 (2001): 139- 145.

3. Warthon-Medina M., et al. "Zinc intake status and indices of cognitive function in adults and children: a systematic review and meta-analysis". European Journal of Clinical Nutrition 69 (2015): 649-661.

4. Abdullah Md., et al. "The role of iron and zinc in cognitive development of children". Asian Journal of Medical and Biological Research 3 (2017): 145-151.

5. Black MM. "The Evidence Linking Zinc Deficiency with Children's Cognitive and Motor Functioning". Journal of Nutrition 133 (2003): 1473-1476.

6. Black MM. "Zinc deficiency and child development". The American Journal of Clinical Nutrition 68 (1998): 464-469. 
7. Umamaheswari K., et al. "Effect of iron and zinc deficiency on short term memory in children". Indian Pediatrics 48 (2001): 289-293.

8. Shashi A., et al. "Linkages of biomarkers of zinc with cognitive performance and taste acuity in adolescent girls". International Journal of Food Sciences and Nutrition 65 (2014): 399-403.

9. Amani R., et al. "Association of cognitive function with nutritional zinc status in adolescent female students". Progress in Nutrition 21 (2019): 86-93.

10. Chiplonkar., et al. "Zinc Supplementation Improved Cognitive Performance and Taste Acuity in Indian Adolescent Girls". Journal of the American College of Nutrition 28 (2009): 388396.

11. Gibson RS., et al. "A growth-limiting, mild zinc-deficiency syndrome in some Southern Ontario boys with low height percentiles". The American Journal of Clinical Nutrition 49 (1989): 1266-1273.

12. Khodashenas E., et al. "The Effect of Zinc Supplementation on Cognitive Performance in Schoolchildren". International Journal of Pediatrics 3 (2015): 1033-1038.

13. Asih SR., et al. "Effects of zinc supplementation on nutritional status and cognition”. Paediatrica Indonesiana 46 (2006): 6470 .

14. Pongcharoen T., et al. "Long-term effects of iron and zinc supplementation during infancy on cognitive function at $9 \mathrm{y}$ of age in northeast Thai children: a follow-up study". The American Journal of Clinical Nutrition 93 (2011): 636-643.

15. Caulfield LE., et al. "Maternal gestational zinc supplementation does not influence multiple aspects of child development at 54 mo of age in Peru". The American Journal of Clinical Nutrition 92 (2010): 130-136.

16. Christian P., et al. "Prenatal micronutrient supplementation and intellectual and motor function in early school-aged children in Nepal”. JAMA 304 (2010): 2716-2723.
17. Tamura T., et al. "Effect of zinc supplementation of pregnant women on the mental and psychomotor development of their children at $5 \mathrm{y}$ of age". The American Journal of Clinical Nutrition 77 (2003): 1512-1516.

\section{Volume 5 Issue 11 November 2021 \\ (C) All rights are reserved by Gomaa F Salma.}

